\title{
Extrapolating Tumor Invasion Margins for Physiologically Determined Radiotherapy Regions
}

\author{
Ender Konukog̃lu ${ }^{1}$, Olivier Clatz ${ }^{1}$, Pierre-Yves Bondiau ${ }^{2}$, \\ Hervé Delingette $^{1}$, and Nicholas Ayache ${ }^{1}$ \\ ${ }^{1}$ Asclepios Research Project, INRIA Sophia Antipolis, France \\ ender.konukoglu@sophia.inria.fr \\ ${ }^{2}$ Centre Antoine Lacassagne, Nice, France
}

\begin{abstract}
In radiotherapy, the constant margin taken around the visible tumor is a very coarse approximation of the invasion margin of cancerous cells. In this article, a new formulation to estimate the invasion margin of a tumor by extrapolating low tumor densities in magnetic resonance images (MRIs) is proposed. The current imaging techniques are able to show parts of the tumor where cancerous cells are dense enough. However, tissue parts containing small number of tumor cells are not enhanced in images. We propose a way to estimate these parts using the tumor mass visible in the image. Our formulation is based on the Fisher-Kolmogorov Equation that is been widely used to model the growth of brain tumors. As a proof of concept, we show some promising preliminary results, which demonstrate the feasibility of the approach.
\end{abstract}

\section{Introduction}

Glial based tumors form the major class of tumors in the central nervous system. Among this class, high grade ones are incurable despite state-of-the-art therapies, and patients have a median survival rate of approximately 1 year, 1 . The diffusive nature of these tumors inhibits the apparent boundary between cancerous and healthy regions, decreasing effects of conventional therapy, like surgical resection and radiotherapy. The current imaging techniques are able to detect only a part of the total tumor, 22. In the literature there are several suggestions for the lowest detection limit of $\mathrm{CT}$ images in terms number of tumor cells per unit volume or area, 2, 3, 4. Based on these suggestions and medical advice, in this work we are using a relative value: $5 \%$ of the maximum number of tumor cells brain paranchyma can hold, as this detection limit. Although up to our knowledge, there is no such a limit suggested for MRI it is the common practice to assume the same limit for T2 weighted images. In Figure 1 we illustrate the visible tumor profile in the image (the solid line curve) and the total tumor profile as given in theory. The usual clinical practice is to surgically remove the visible part and try to kill tumor cells invisible in the images, using radiotherapy and/or chemotherapy. The conventional radiotherapy treatment 

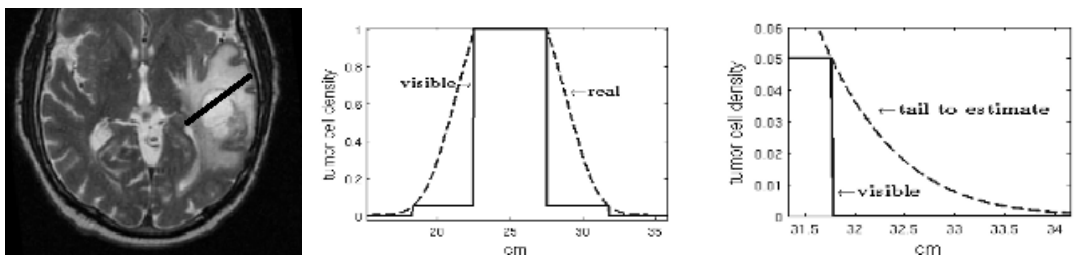

Fig. 1. Left: T2-weighted MR image with the tumor, Middle: Visible tumor density profile for the black line of the left image is given in solid curve and the theoretical tumor density profile shown by the dashed curve, Right: Regions with less tumor density than $5 \%$ are not visible in the image. The aim of this paper is to estimate cell density distribution in these regions (dashed curve).

uses a geometric approach and applies radiation in a constant margin of about $2 \mathrm{~cm}$, around the surgical site. This approach does not take into account different speed of tumor diffusion for the grey and the white matter, 5]. Using the same margin for both matters may kill healthy grey tissue while not reaching the extent of tumor cells in the white matter, leaving alive cancerous cells on the fibers. These cells would cause reccurence of the tumor in time. By constructing physiologically determined radiotherapy margin, which takes into account tumor growth dynamics and the properties of the tissue, the therapy can be more effective.

Mathematical modeling of tumor growth dynamics gives us a better insight on the physiology of this process and it could be used to improve the treatment planning (surgery, radiotherapy or chemotherapy). There is a lot of work on modelling the growth of high grade gliomas. Approaches taken to solve this problem can be coarsely classified into two groups, microscopic and macroscopic ones. Microscopic ones try to describe cell divisions and invasion by means of interactions between tumor cells and their surrounding tissue, 6, 7. On the other hand, macroscopic models describe the evolution of local tumor cell densities and try to capture the dynamics by general equations and with fewer parameters. Moreover, such models may more easily be adapted to act on real medical images. Most of the macroscopic models, [1, 3, 4, are based on the reactiondiffusion formalism introduced by Murray in [8, 1]. This formalism uses the general type of PDEs called the reaction-diffusion to model the tumor growth dynamics.

We are proposing a method to estimate the distribution of tumor cells for low density regions using a single image with a set of estimated parameters. Our formulation constructs an approximation to the tails of the tumor distribution (invasion margins) which are not visible in images. In addition, this formulation creates appropriate initial conditions for tumor growth models, which can be used for simulating tumor growth and predicting the distribution of the tumor in a future time. Using an initial condition with well estimated tails will increase ability of these models to predict tails of tumor as well as its whole distribution. 


\section{Method}

\subsection{Fisher-Kolmogorov Equation}

In this article, we assume that tumor growth dynamics obey a reaction-diffusion Equation similar to the ones proposed in [3, 4, in which diffusion of tumor cells is correlated with the diffusion of water, hence with the DT-MRI. The specific Equation we will use is the Fisher-Kolmogorov (F-KPP) Equation given as:

$$
\begin{array}{r}
\frac{\partial u}{\partial t}=\nabla \cdot(D(\mathbf{x}) \nabla u)+\rho u(1-u) \\
D \nabla u \cdot \vec{n}_{\Sigma}=0
\end{array}
$$

where $u$ is the normalized tumor cell density between $[0,1]$ (normalized cell densities will be used throughout this article), $D$ represents the diffusion tensor of tumor cells and $\rho$ corresponds to the proliferation rate of tumor cells. In Equation 1, the first term of the right hand-side describes the diffusion of tumor cells towards the tissue, which is governed by $D$. The second term, $\rho u(1-u)$, is the so called reaction term, and it describes proliferation of tumor cells as a logistic growth. There are different types of reaction terms that can be used like the gompertz growth model, $\rho u \ln (1 / u)$, or exponential growth model, $\rho u$. Equation 2 represents the no-flux boundary condition, where $\Sigma$ is the boundary of the brain (ventricles and the skull) and $\vec{n}_{\Sigma}$ is the normal to the boundary. This condition describes the fact that tumor cells do not diffuse through ventricles and through the skull.

The simple version of this Equation in $1 \mathrm{D}$ with constant coefficients and no boundary conditions was first proposed by Fisher, 9], to model the spread of a favoured gene in a population:

$$
\frac{\partial u}{\partial t}=d \frac{\partial^{2} u}{\partial x^{2}}+\rho u(1-u)
$$

where $d$ is the diffusion coefficient. The travelling wave solutions of Equation 3 have been studied throughly by Kolmogorov. Later on Aronson et al. have extended this analysis to the infinite cylinder and studied the travelling plane solutions in [10].

Here we wish to summarize some relevant results in these works. At large times, Equation 3 admits a travelling wave solution in the infinite cylinder. In other words, when the change of $u$ is non-zero in only one direction, $\mathbf{n}$, for very large times the solution can be given in the form:

$$
u(\mathbf{x}, t)=u(\mathbf{n} \cdot \mathbf{x}-c t)=u(\bar{\xi}) \text { as } t \rightarrow \infty
$$

where $c$ corresponds to the speed of the front and $\bar{\xi}=(\mathbf{x} \cdot \mathbf{n}-c t)$ is the moving frame. The speed of the front is a constant, which depends on coefficients $D$ and $\rho$, and also on the tails of the initial condition $u(\mathbf{x}, 0)$. When the initial condition has a compact support the speed of the travelling wave can be given as, 10]:

$$
c=2 \sqrt{\rho \mathbf{n} \cdot(D \mathbf{n})}
$$




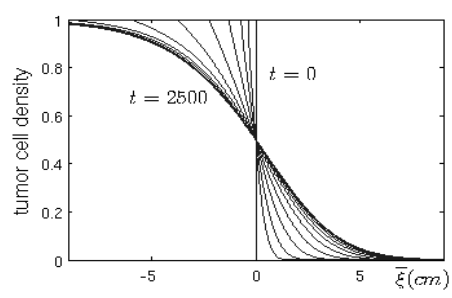

(a)

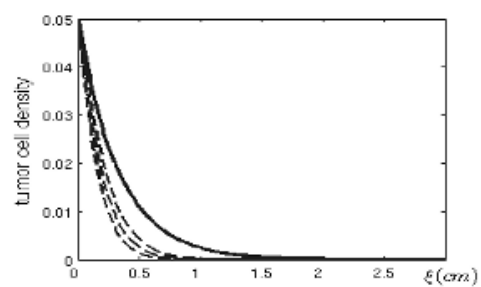

(b)

Fig. 2. (a) Shape of the wavefront is plotted for different time values (0-2500 days) in the moving frame. The profile converges in time. (b) The approximated tail using Equation 8 (solid curve) and actual tails of the wave front at $t=60,90,125$ days (from left to right). As time increases the solid curve approximates the actual tail better.

The planar initial condition with compact support converges to a travelling plane with speed $c$ in time. As the speed of the travelling plane converges to $c$ the shape of it also converges to a constant shape, which does not have an analytical form. Figure 2(a) illustrates the convergence of the front shape both for whole front and for the low density parts.

\subsection{Tumor Tail Extrapolation}

Our aim in this article is to extrapolate the low tumor density regions, invisible in the image, from the visible part of the tumor, at a given time instant. We are going to use the fact that the front shape is constant for the travelling plane solution for large times to extrapolate the low density regions.

Since we are trying to extrapolate the tails (low values of $\mathrm{u}$ ) we propose to linearize the nonlinear reaction term in Equation 1 around $u=0$ and assume $1-u$ can be well approximated by 1 :

$$
\frac{\partial u}{\partial t}=D \Delta u+\rho u
$$

This Equation also admits a travelling wave solution in the infinite cylinder, with the speed $c$ given in Equation 5. The travelling wave solution for this Equation has an analytical solution, and for the low tumor density parts the solution can be given as:

$$
u(\mathbf{x}, t)=u(\bar{\xi}) \cong A e^{-\lambda \bar{\xi}} \text { for } \lambda=-\sqrt{\rho /(\mathbf{n} \cdot(D \mathbf{n}))}
$$

where $A$ is an integration constant.

The value of $\bar{\xi}$ for a point corresponds to the distance of it from the inflection point of the front shape, which is at $u=0.5$ (see Figure 2(a)). However, the only information we get from images is the location of the last visible iso-density contour $u_{0}$, which we assume to be equal to 0.05 , in terms of normalized tumor densities, see Figure 1] Applying the solution given in Equation 7 for the value $u_{0}$, we find $A=u_{0} e^{\lambda \bar{\xi}_{0}}$ where $\bar{\xi}_{0}=\left\{\bar{\xi} \mid u(\bar{\xi})=u_{0}\right\}$. Placing this in Equation 7 


$$
u(\mathbf{x}, t) \cong u_{0} e^{-\lambda\left(\bar{\xi}-\bar{\xi}_{0}\right)}=u_{0} e^{-\lambda \xi}
$$

where $\xi=\bar{\xi}-\bar{\xi}_{0}$, which is the distance of a point from the iso-density surface $u=u_{0}$. Unlike $\bar{\xi}, \xi$ is observable in the images since the $u_{0}$ iso-density contour is visible. In Figure 2(b) we plot the tail approximation given by Equation 8 as a function of $\xi$ together with the front shape of the travelling plane taken at different time instants.

This approximation is only valid when the diffusion tensor is constant over the whole domain and the motion is only in one direction, which is not the case. Moreover when the tumor front is curved, its motion is not in one direction and the solution cannot be given in terms of travelling wave. However, to compute the tumor cell densities at tails in MR images we make the following hypothesis: $D$ is constant and the motion of the front is only in one direction within a voxel. Based on this assumption we construct the solution given in Equation 7 for each voxel. The direction of motion and the initial value for each voxel are defined by adjacent voxels. The 1-dimensional illustration of this idea is given in Figure 3 . Equation 7 is the integral solution of $\partial u / \partial \mathbf{n}=(\lambda \xi) u$, since $\xi$ direction is normal

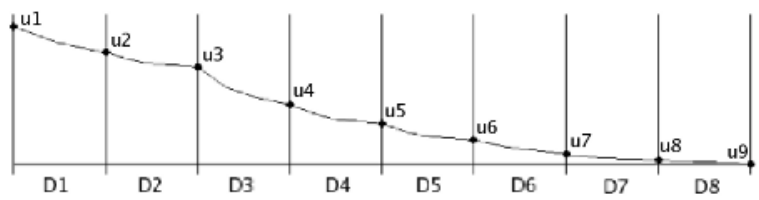

Fig. 3. The formulation constructs the solution at grid point $p+1$ using the $D_{p}$ as the diffusion tensor and $u_{p}$ as the initial value

to the front. Replacing $\mathbf{n}$ with $\nabla u /|\nabla u|$ in this Equation, we obtain the following static Hamilton-Jacobi Equation that constructs the solution given by Equation 7 at each voxel as shown in Figure 3.

$$
\frac{\sqrt{\nabla u \cdot(D \nabla u)}}{\sqrt{\rho} u}=1, u(\Gamma)=u_{0}
$$

where $\Gamma$ is the last visible iso-density contour of the tumor in the image. This Equation has two solutions at each point, one with increasing $u$ and one with decreasing $u$. Since the F-KPP Equation tells us that as we move away from the visible contour, the values of $u$ will decrease, for all points we choose the decreasing solution. Using Equation 9 we start from $\Gamma$ and move outwards as we find $u$ values for each point. We also have to include the no-flux boundary condition given in Equation 1 in this approximation. Let $\Omega$ be the whole image domain and $\Omega_{B}$ be ventricles and the skull with its exterior. We enforce the no-flux boundary condition by setting the domain of Equation 9 as $\Omega_{s}=\Omega / \overline{\Omega_{B}}$. In which $\overline{\Omega_{B}}$ is the closure of $\Omega_{B}$. 


\subsection{Numerical Implementation}

There are several numerical techniques proposed to solve static H-J Equations, amongst which sweeping methods, such as fast marching, are the most popular. However, we do not use the sweeping methods because they construct the integral solution at the center of each voxel, for which the accuracy is low when the resolution of the image is low. In order to solve Equation 9 numerically we embed the static H-J Equation into a dynamic one using the method proposed by Osher in [11. This methodology allows us to make use of the level set idea and obtain subvoxel accuracy. This is crucial since the resolution of MR images is not high $(1 \mathrm{~mm} \times 1 \mathrm{~mm} \times 4 \mathrm{~mm})$. The embedding is done by placing each isodensity contour of $u$ as the zero-level set of a higher dimensional function $v$. Namely, the embedding is such that, $u(\xi)=\tau$ iso-density contour corresponds to the $v(\xi, \tau)=0$. Using this we transform Equation 9 into Equation 10 .

$$
\frac{\partial v(\xi, \tau)}{\partial \tau}=\frac{\sqrt{\nabla v \cdot(D \nabla v)}}{\sqrt{\rho} \tau} \text { for } \tau \in\left(0, u_{0}\right]
$$

where $\tau$ decreases at every iteration so that we find lower tumor density regions. As the zero level-set of $v$ moves over the domain of $u, \Omega_{s}$, for different $\tau$ we construct the $u$ value at each point as $u(\xi)=\tau$ for $\xi \mid v(\xi, \tau)=0$. We have used the method proposed by Bryson et al. in [12] to solve this dynamic H-J Equation numerically in $3 \mathrm{D}$, due to its ease of implementation and accuracy properties. Using an adaptive $\tau$ step as: $\Delta \tau=\tau / 30$ the algorithm takes around 30 seconds to compute the tails in the whole brain until $u=0.0005$.

As of implementing Equation 1 in 3D, we have used finite differences with a semi-implicit solver (pre-conditioned conjugate gradient). The boundary conditions for the diffusion part given in Equation 2 were included in the numerical scheme by using the flux method proposed by McCorquodale et al. in 13.

\section{$3 \quad$ Results}

We have run our experiments by artifically placing tumors on T2-weighted images taken from healthy subjects. We assume Equation 1 models the tumor growth accurately, so we compare our results using a synthetic tumor grown with this model, for which we know the whole distribution. Giese et al. in [5] have shown in-vitro that tumor cells move faster on myelin sheath. We use this information and a correlation between water and tumor diffusion to construct our tumor diffusion tensor, $D$, from DT-MR images of subjects as done in [3,4]. We use $D_{\text {grey }}=d_{0} \mathbf{I}$, where $\mathbf{I}$ is the identity matrix, creating isotropic diffusion tensor $D_{\text {grey }}$ for the grey matter. $D_{\text {white }}=d_{0} \alpha D_{\text {water }}$ is the diffusion tensor for tumor cells in the white matter, which has the same orientation as the normalized water diffusion $D_{\text {water }}$ at that point. $\alpha$ describes the speed difference of tumor cells in the white and the grey matter. In our experiments we have taken $\alpha$ so that the largest diffusion in the white matter would be equal to 50 times 

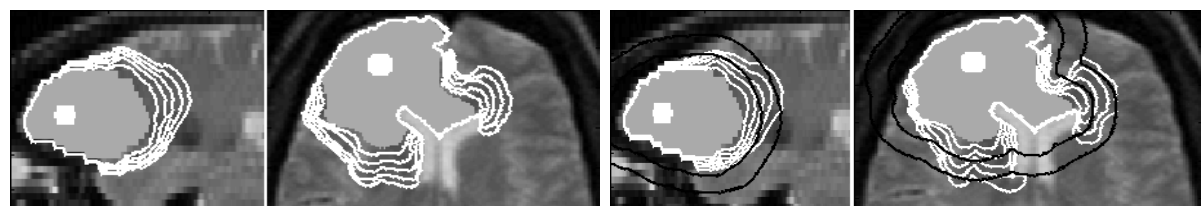

Fig. 4. Left: Sagital and axial views of the T2-weighted image for the synthetic tumor including its iso-density contours from 0.05 to 0.003. Right: Sagital and axial views of the same T2-weighted image with estimated iso-density contours based on our method. Black contours correspond to constant margin radiotherapy regions for $1 \mathrm{~cm}$ and $2 \mathrm{~cm}$.

the isotropic diffusion in the grey matter. The two parameters for the formulation, $d_{0}$ and $\rho$ were set using the values proposed in [1] as $0.0013 \mathrm{~cm}^{2} / d a y$ and 0.012 cells/day respectively.

This experiment shows the ability of the proposed model in constructing low tumor density regions from a single image. For this, we first grew a synthetic tumor for 180 days. Then we applied the 5\% detection threshold and created the T2-weighted image with the synthetic tumor. In Figure 4 on the left we show the saggital and the axial slices of the T2-weighted image with some isodensity contours below the detection threshold. In the same figure on the right we show same images with corresponding iso-density contours extrapolated by the H-J formulation. In both sagital and axial views, we see that the extrapolated tumor tails using H-J formulation are in very good agreement with tails of the synthetic tumor. We can conclude that in case of a synthetic tumor grown by the F-KPP model, given an image and a set of estimated parameters $(D$ and $\rho)$, the proposed formulation is successful in extrapolating the low tumor density parts, which are undetectable by current imaging techniques. We also plotted the conventional radiotherapy regions (in black) on the image with extrapolated tumor tails to show the difference between the constant margin approach and the estimated invasion regions. Observe that by using physiologically determined radiotherapy margin instead of a constant one, more tumor cells can be killed while less healthy tissue is harmed.

\section{Discussion}

We have proposed a formulation to estimate low tumor density regions in a given MR image, which are undetectable by current imaging techniques, provided a set of parameters $\{D, \rho\}$ either manually adjusted or estimated by the radiotherapist. Our formulation is based on the F-KPP growth model, which is been widely used for simulating tumor growth in medical images. As a proof of concept we have demonstrated the ability of this formulation in constructing tumor tails by comparing it with a synthetic tumor. Based on these results, we conclude that our formulation is successful in using the underlying tissue structure and the dependence of low density regions to the visible part, for extrapolating undetectable parts of the tumor. These promising preliminary results demonstrate the feasibility of this approach for real patient images. 

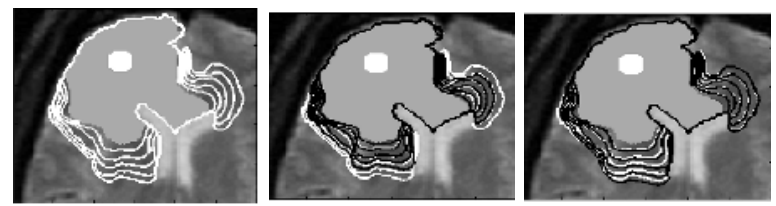

Fig. 5. Left: Undetectable tails of the synthetic tumor are plotted (white). Middle: Contours predicted by F-KPP model (black) using a simple step-edge initialization with actual contours (white). Right: Contours predicted using our tumor tail estimation as an initial condition with actual contours (white).

This formulation constructs an estimate for the undetectable part of the tumor in images, showing the invasion margin, which can be used to determine radiotherapy regions based on tumor growth dynamics. It also provides medical doctors a tool to observe the effect of parameters, like speed of the tumor front and proliferation rate, on undetectable parts of the tumor. Moreover, it constructs an appropriate initial condition for tumor growth models, which can be used for predicting the future distribution of the tumor. Since the initial condition is the key to predict future tumor densities and tail distributions influence these values, using an initial condition with good tail estimates will increase the accuracy of prediction. In Figure 5, we demonstrate the ability of our formulation in creating appropriate initial conditions for the prediction of future tumor cell density. Indeed, we compare two predictions at day 180 (in black) using the F-KPP model based on the knowledge of the visible tumor at day 90. In the middle image, a simple step-edge initialization while in the right image our tumor tail estimation is used. We observe that the prediction starting with an initial condition with appropriate tails is much more accurate in the case of a synthetic tumor grown by the F-KPP model.

The ability of the proposed formulation has been demonstrated using synthetic tumors. We are currently working on validating the performance of the formulation in images with histo-pathological data. Besides that, automatic estimation of model parameters, like $D$ and $\rho$, from images taken at different time instances is an open problem we are working on.

\section{References}

1. Swanson, K., Alvord, E., Murray, J.: Virtual brain tumours (gliomas) enhance the reality of medical imaging and highlight inadequacies of current therapy. British Journal of Cancer $\mathbf{8 6}$ (2002)

2. Tracqui, P., Cruywagen, G., Woodward, D., Bartoo, G., Murray, J., Alvord, E.: A mathematical model of glioma growth: the effect of chemotherapy on spatiotemporal growth. Cell Proliferation 28(1) (1995)

3. Clatz, O., Sermesant, M., Bondiau, P., Delingette, H., Warfield, S., Malandain, G., Ayache, N.: Realistic simulation of the $3 \mathrm{~d}$ growth of brain tumors in $\mathrm{mr}$ images coupling diffusion with biomechanical deformation. IEEE T.M.I. 24(10) (2005) 
4. Jbabdi, S., Mandonnet, E., Duffau, H., Capelle, L., Swanson, K., Pélégrini-Issac, M., Guillevin, R., Benali, H.: Simulation of anisotropic growth of low-grade gliomas using diffusion tensor imaging. Magnetic Reson. in Med. 54 (2005)

5. Giese, A., Kluwe, L., Laube, B., Meissner, H., Berens, M., Westphal, M.: Migration of human glioma cells on myelin. Neurosurgery 38(4) (1996)

6. Cristini, V., Lowengrub, J., Nie, Q.: Nonlinear simulation of tumor growth. Journal of Math. Biol. 46 (2003)

7. Patel, A., Gawlinski, E., Lemieux, S., Gatenby, R.: A cellular automaton model of early tumor growth and invasion. Journal of Theo. Biol. 213 (2001)

8. Murray, J.: Mathematical Biology. Springer-Verlag (2002)

9. Fisher, R.: The wave of advance of advantegous genes. Ann. Eug. 7 (1937)

10. Aronson, D., Weinberger, H.: Multidimensional nonlinear diffusion arising in population genetics. Advances in Mathematics 30 (1978)

11. Osher, S.: A level set formulation for the solution of the dirichlet problem for hamilton-jacobi equations. SIAM J. Math. Anal. 24(5) (1993)

12. Bryson, S., Levy, D.: Central schemes for multidimensional hamilton-jacobi equations. SIAM J. Sci. Comput. 25 (2003)

13. McCorquodale, P., Colella, P., Johansen, H.: A cartesian grid embedded boundary method for the heat equation in irregular domains. J. Comp. Phys. 173(2) (2001) 